\title{
Comparison of Digital Image Analysis and Conventional Microscopy In Evaluating Erythrocyte Morphology in Peripheral Blood Smears*
}

Erick Martin Yturralde, Karen Bulseco-Damian, Nelson Geraldino

Department of Laboratories, Philippine General Hospital, University of the Philippines-Manila

\section{ABSTRACT}

Background and Objectives. The use of conventional microscopy still forms the basis for the morphologic evaluation of erythrocytes despite widespread use of automated tests in the hematology laboratory. This requires a considerable length of time and expertise, and have the potential of becoming a source of errors and delay in reporting. Advances in image processing and machine learning in recent years have shown acceptable performance characteristics and have promising applications in the diagnostic laboratory. Use of these newly-developed technologies can address the stated problems and provide an alternative approach in the microscopic analysis of erythrocytes.

Methodology. This prospective validation study compared digital image analysis using a machinelearning based image recognition algorithm with conventional microscopy performed by a trained microscopist, which served as the reference standard. Random deidentified anticoagulated peripheral blood samples submitted to the hematology laboratory were assessed.

Results. A total of 956 erythrocytes were evaluated after image processing using support vector machine and routine microscopy as classifiers of erythrocytes into three categories: size, central pallor, and shape. The tested software was able to achieve a strong level of agreement compared to conventional microscopy, having kappa values ranging from 0.81 to 0.86 . Accuracy for size, central pallor and shape were $89.88 \%, 93.72 \%$ and $87.89 \%$, respectively.

Conclusion. The validated image recognition software is an acceptable diagnostic test in determining erythrocyte morphology in peripheral blood smears. Its integration can potentially minimize hands-on time and improve the diagnostic laboratory workflow.

Registration. Philippine Health Research Registry (PHRR) ID: PHRR191211-002348; University of the Philippines Manila Research Ethics Board (UPMREB): 2019-356-01

Key words: erythrocyte morphology, digital imaging, microscopy

ISSN 2507-8364 (Online)

Printed in the Philippines.

Copyright $(2020$ by the PJP.

Received: 17 December 2019

Accepted: 18 February 2020.

Published online first: 22 March 2020.

https://doi.org/10.21141/PJP.2020.02

Corresponding author: Erick Martin Yturralde

E-mail:ehyturralde@up.edu.ph

* Presented in the $25^{\text {th }}$ Pathology Resident's Research Forum held in the Philippine General Hospital, on the $8^{\text {th }}$ of November 2019.

\section{INTRODUCTION}

\section{Background}

The complete blood count (CBC) is one of the most commonly requested tests in a diagnostic laboratory, providing the clinician a reflection of the cellular constituents of blood which includes red blood cell (RBC), white blood cell (WBC) and platelet counts, as well as, red cell indices and white cell differential counts. With the advent of laboratory automation, the CBC result can be released by a hematology laboratory in a relatively rapid and reliable manner leading to faster turn-around times and specimen reporting. Depending on the manufacturer, these hematology analyzers (Beckman Coulter Hematology Analyzer, Siemen's ADVIA®, Sysmex Hematology Analyzer, and Abbott CELL-DYN Sapphire®) rely on the principles of electrical impedance and light scatter, with the conventional microscopy in peripheral blood smears still forming the basis in erythrocyte morphologic classification. ${ }^{1}$ In high-volume settings, the use of peripheral blood smears remains a laborious and time-consuming process that can take 
several minutes from slide preparation to microscopic reading. In smaller laboratories handling relatively smaller number of specimens, the lack of expertise of the microscopist can be an attributable factor to incomplete or inaccurate results. ${ }^{2}$ With the considerable reliance of clinical hematology in diagnostic laboratory procedures for establishing a diagnosis, the need for accurate and timely results are of utmost priority.

As automated image recognition of cellular elements started becoming more accessible, the advantages of its application in the diagnostic field became apparent, from minimizing technologist hands-on time to improving work flow and decreasing turn-around time of specimens. In recent years, the rapid advance of artificial intelligence, specifically machine learning, provided benefits in data processing by focusing on the desired input-output behavior rather than manually coding for the desired response for all possible inputs. The image recognition software used in analyzing urine sediments is a good example of the latter, relying on a sizeable database provided by the equipment manufacturer. Depending on the machine learning algorithm used, the program can be 'trained' into appropriately classifying an input data into a desired output classifier. Applying this in the field of diagnostic hematology laboratory, an input data can be represented as captured microscopic images of red cells and the output classifier being represented by the size, pallor and shape characteristics. By providing the algorithm multiple images of, for example, echinocytes, the software, in theory, will be able to correctly identify other red cells with the same morphology.

\section{Objectives of the Study}

With the above presented concepts in mind, the present study aimed to prospectively validate a newly developed image recognition software that utilized machine learning algorithm in the classification of erythrocyte morphology and compared it to conventional microscopy performed by a trained microscopist. The specific objectives were as follows: 1) Using photomicrographs of erythrocytes, the cells were classified according to its morphologic characteristic (size of cell, area of central pallor and shape of cell) by a microscopist, which served as the reference standard for the purposes of the study, 2) the same images were classified by the image recognition software, 3) statistical agreement between the two methods was determined using kappa statistics, 4) sensitivity, specificity and accuracy of the image recognition software were calculated.

\section{Significance of the Study}

This study aims to provide a novel method in the identification of erythrocyte morphology in the diagnostic clinical laboratory and if proven as an acceptable alternative, will be beneficial to the following groups:

Pathologists. The results of this study will expand the knowledge of pathologists regarding the potential applications, advantages and inherent limitations of machine learning in the laboratory. This knowledge could help pathologists provide relatively accurate results with minimal subjectivity and improved turn-around times.
Medical Technologists. The results of this study can improve the test process by minimizing hands-on time in handling specimens, allowing the technologist to focus on other important aspects of the laboratory workflow. The study can also provide new knowledge regarding how machine-learning based algorithms can be applied in a diagnostic laboratory.

Clinicians and Patients. The expected improved turnaround time gained from the method can provide clinicians time-sensitive laboratory information and aid them in formulating treatment plans that can ultimately benefit the patient.

\section{Review of Related Literature}

Ever since the microscope was introduced in the field of health sciences, the microscopic study of blood elements has brought about several observations in the characteristics of erythrocytes, from the size of the cell, central pallor, and alterations in its shape. Through various observations, several disease states were correlated with the characteristic morphologic alteration of erythrocytes. ${ }^{3,4}$ In the 1930 s, Wintrobe introduced the evaluation of red cell indices such as Mean cell volume (MCV), mean corpuscular hemoglobin (MCH), and mean corpuscular hemoglobin concentration (MCHC), which to this day are still used in the evaluation of erythrocytes. ${ }^{5}$ In many forms, these elements of the complete blood count have been adopted in the processes employed by automated hematology analyzers. However, the practice of evaluating peripheral blood smears for identifying erythrocyte morphology has endured despite the widespread use of automated analyzers. ${ }^{2,6}$

Currently, the need to perform peripheral blood smears in the evaluation of erythrocytes after using automated analyzers are set by individual laboratories and employ specific reporting methods and terminologies. ${ }^{7,8}$ Reasons as to why automated analyzers are currently unable to characterize red cell morphology has highlighted their limitations, specifically: 1) the analytical principles employed by automated hematology analyzers such as cell impedance and light scattering are physically unable to identify the specific red cell morphologies, 2) the methods employed intentionally alter the red cells as they pass through a measuring aperture used to calculate the cell volume, 3) the use of specialized procedures (three-dimensional optical measurement of erythrocytes, detection of high oxygen affinity hemoglobin) requires highly specialized equipment and reagents that are not readily available and uneconomical as part of routine screening, and compared to these methods, the manual peripheral blood smears are more feasible as a screening or adjunct method in specimens flagged by automated analyzers. ${ }^{4,9}$

The persistence of the manual counting and evaluation of the peripheral smears requires a significant amount of hands-on time, making it predisposed to interobserver variability and bias. The additional use of laboratory resources and the possible consequences in the test accuracy can lead to prolonged test reporting. ${ }^{6,8}$ 
The rapid development of machine learning algorithm as reflected by the number of published papers in the last decade has provided several glimpses on its application in laboratory medicine. ${ }^{10-12}$ Machine learning is different from the so-called "expert systems" that are commercialized for specific laboratory use. The latter follow predefined lines of code that state specific parameters, and by following logic rules, the system checks if the specimen characteristics fall into which set parameter. ${ }^{13}$ This rigid approach is vastly different from machine learning, where the parameters are "learned" by only providing input and output information. Laboratory parameters and data are ripe for application of machine learning systems because of the use of quantitative data that are relatively easy to categorize or coded. With the growing complexity and amount of data produced by the laboratory, machine learning systems can provide immediate feedback not only to the healthcare team, but to the patient as well. ${ }^{13-15}$

Computer software-driven morphological cell analysis relies on mathematical algorithms to identify cellular features. It employs several processing techniques such as edge detection, image enhancement, skeletonization and pattern recognition to produce a geometric figure that can be analyzed. ${ }^{16}$ With the use of machine learning, these set of complex data can yield better performance in resolving the task of cellular morphological analysis. Support vector machine, one of the models of machine learning, utilizes supervised learning methods wherein the algorithm categorizes a set of training examples. It is able to resolve binary problems by maximizing margins of errors to make hyperplanes from support vectors and handle discriminatory functions. ${ }^{16,17}$ By providing several examples, a decision boundary and model is formed able to predict new examples into its category. Appropriate tuning of the model is performed by providing additional training examples and regression from erroneous results. The inputs from the dataset are known while the outputs are learned in the form of responses. These methods can increase reliability and accuracy, making its utilization more acceptable in the diagnostic laboratory. ${ }^{18}$

\section{METHODOLOGY}

\section{Ethical Considerations}

The study was accomplished after seeking ethical approval from the University of the Philippines Manila Research Ethics Board (UPMREB). A waiver of consent was requested and approved as there were no risks to the study participants. All peripheral blood specimens and smears analyzed in the study were deidentified to ensure anonymity and confidentiality of the patients during data collection, specimen handling and storage.

\section{Study Design}

The study design used a validation of a novel assay platform in the form of machine learning-based algorithm in comparison with the conventional microscopic method used in erythrocyte morphologic assessment. Figure 1 summarizes the study methodology. Both procedures involved the evaluation of peripheral blood smears presenting with various erythrocyte parameters in accordance with the inclusion criteria stated below.

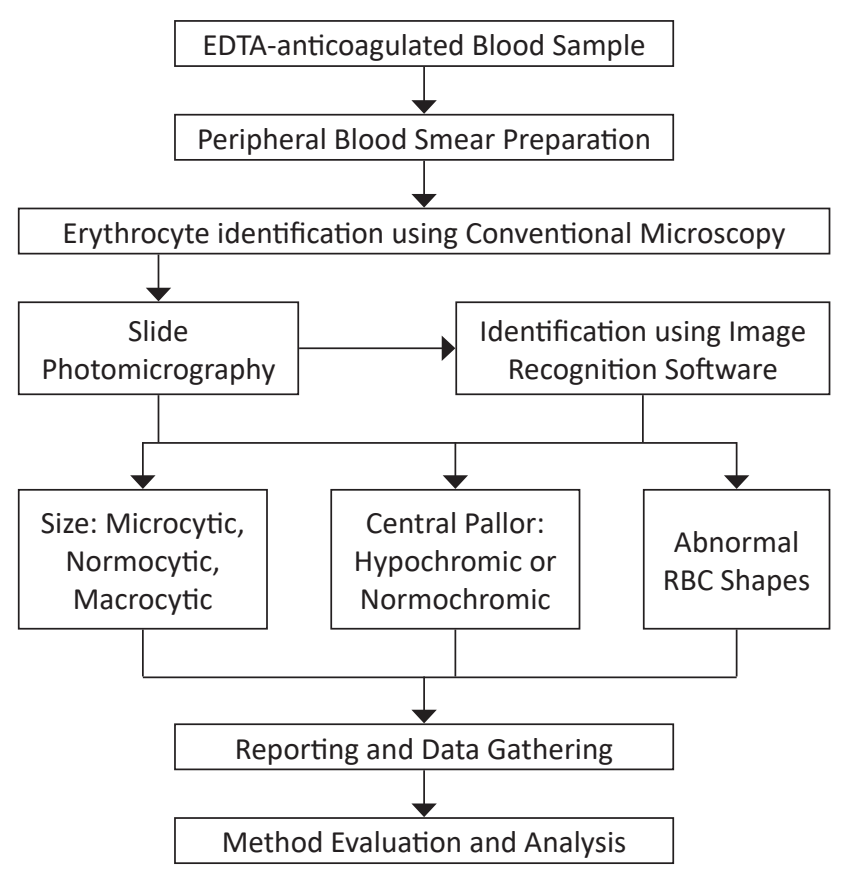

Figure 1. Study workflow diagram.

\section{Sample Size}

Study sample sizes were computed based on a test agreement between two raters using kappa statistic to detect a true kappa value of at least 0.80 (almost perfect agreement). The sample sizes are computed based on three (3) categories: for erythrocyte chromicity or central pallor, requiring at least 172 subjects, for erythrocyte size, requiring at least 144 subjects and erythrocyte shape, requiring 135 subjects. These power calculations are based on a significance level of $95 \%$. Overall, this study needed at least 172 subjects. Random samples from the hematology laboratory were collected within two months (October to November 2019).

\section{Inclusion Criteria}

1) Ethylenediaminetetraacetic acid (EDTA)-anticoagulated blood from patients submitted to the section of Hematology for complete blood count with normal red cell counts and red cell indices.

2) Submitted EDTA-anticoagulated blood from patients for complete blood count and flagged by the hematology analyzer due to abnormal red cell counts or cell indices, requiring peripheral blood smears.

3) Submitted EDTA-anticoagulated blood from patients for complete blood count with physician-requested peripheral blood smear.

\section{Exclusion Criteria}

1) Blood from patients flagged due to improper specimen collection or presence of blood clots.

2) Blood from patients with hyperleukocytosis or other conditions presenting with inclusions and intracellular parasites which might impair red cell visualization.

\section{Data collection}

Cases were collected prospectively from EDTAanticoagulated patient blood samples submitted to the section of Hematology submitted from October to 
November 2019. Blood smears were manually prepared and generated by placing a small drop of blood on one end of a clean glass slide and smeared using a spreader slide. The smear is then allowed to air-dry and stained with Wright-Giemsa stain in strict accordance with laboratory protocols to maintain standardization among all smears. No patient data or clinical information were collected in the study.

Each blood smear was evaluated by a single trained microscopist, who is an experienced medical technologist working in the hematology laboratory, and photomicrographs of the identified red cells were captured using a camera (Raspberry Pi camera module) attached to the eyepiece of a microscope (Olympus CX23) under the oil immersion objective (100X magnification). The field of the smear where the erythrocytes will be optimally evaluated will be initially identified by the microscopist and images will be taken. Depending on the number of optimal fields present, five to ten images were taken for each smear. The camera attachment is directly interfaced with a single board computer (Raspberry Pi) with LCD monitor which also contains the image recognition software (Photograph 1). The same identified cells in the photomicrograph will then be evaluated using an image recognition software as developed by Divina \& Felices using Python OpenCV. The software utilized several image processing techniques (Photograph 2) and formed a machine learning
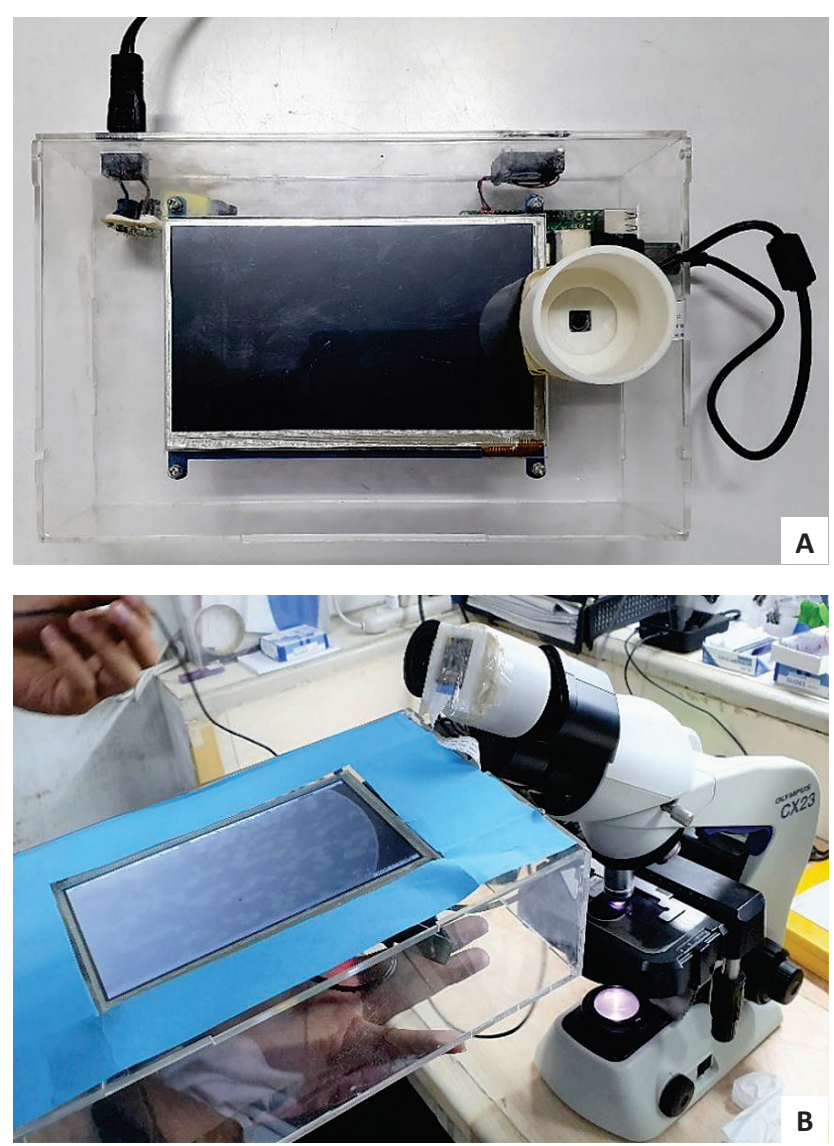

Photograph 1. Prototype with camera attachment for the microscope (A) and prototype with microscope set-up during photomicrography (B). model using Support vector machine in classifying erythrocytes as shown by the flow chart below (Figure 2). ${ }^{19}$ The microscopist and computer engineer operating the image analyzer were blinded to the results of the other group.

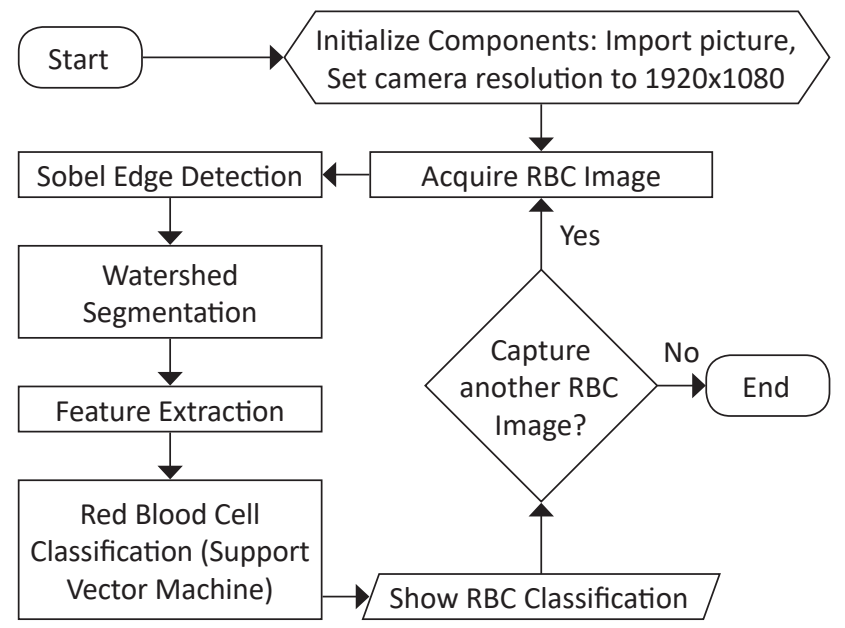

Figure 2. Image recognition software process flow.

RBC morphologies were classified by the microscopist and the image analysis software as to RBC size (normocyte, microcyte, and macrocyte), central pallor (hypochromic and normochromic), and RBC shape (normal, dacrocytes, echinocytes, elliptocytes, spherocytes, stomatocytes, and target cells). All data generated by the software are automatically compiled in a CSV file format (Microsoft ${ }^{\circledR}$ Office Excel ${ }^{\circledR}$ ) for future reference.

\section{Data analysis}

The erythrocyte findings of the microscopist were considered the "true" value for the purposes of the study. Three confusion matrix tables were generated for each RBC classification (central pallor, size and shape) to demonstrate the performance of the image recognition software while the determination of the reliability between the two modalities was analyzed using Cohen's kappa. This is a widely used method in the evaluation of machine learning-based systems. The kappa statistic guidelines established by $\mathrm{McHugh}^{20}$ was used in the interpretation of the values as seen in Table 1. Accuracy, sensitivity (true positive rate or recall) and specificity (true negative rate) were also computed from the generated confusion matrices. The $95 \%$ confidence intervals (CI) for sensitivity and specificity were computed using "exact" Clopper-Pearson confidence intervals.

\begin{tabular}{|c|c|c|}
\hline Value of Kappa & Level of Agreement & Percentage of Data that are Reliable \\
\hline $0-0.20$ & None & $0-4 \%$ \\
\hline $0.21-0.39$ & Minimal & $4-15 \%$ \\
\hline $0.40-0.59$ & Weak & $15-35 \%$ \\
\hline $0.60-0.79$ & Moderate & $35-63 \%$ \\
\hline $0.80-0.90$ & Strong & $64-81 \%$ \\
\hline Above 0.90 & Almost Perfect & $82-100 \%$ \\
\hline
\end{tabular}




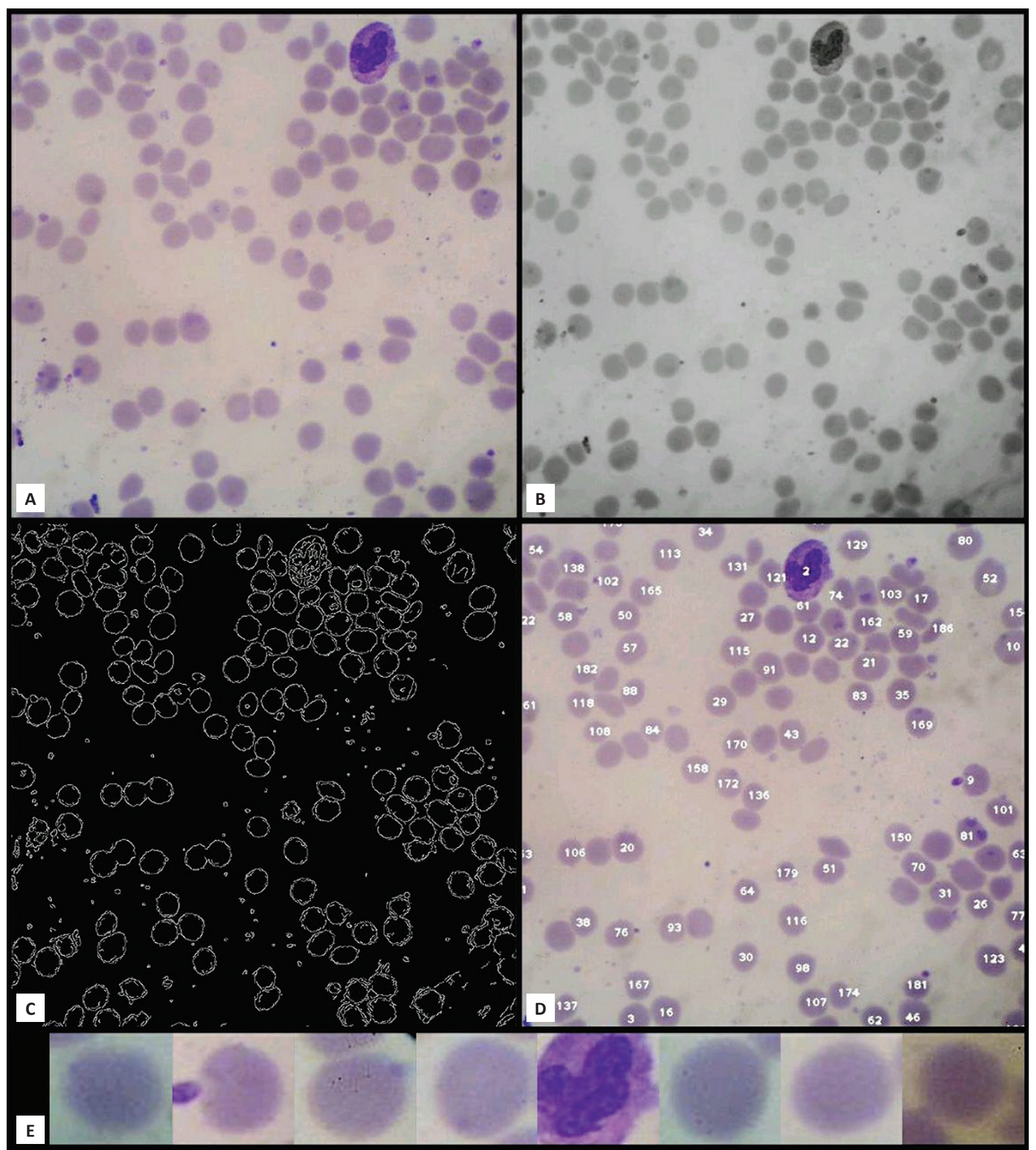

Photograph 2. Image processing algorithm utilized by the software. Shown here are the original image (A), watershed segmentation (B), Sobel edge detection (C), output after image processing (D) and sample of cropped cells identified by the software $(\mathrm{E})$.

\section{RESULTS}

The image recognition software was able to effectively isolate and identify each red blood cell (RBC) according to size, central pallor and shape. For classification, the software identified seven features (area, perimeter, diameter, shape geometric factor, deviation value, central pallor and target flag) and analyzed the image using the machine learning algorithm based on acquired patterns from the training set. Based on these features and the training datasets, a confidence value will be assigned by the software as to how definite is its classification on a particular erythrocyte. A confidence value of at least 70 is sufficient for the software to assign a specific classification. The images used for the machine learning algorithm training datasets were acquired from a different set of cases in relation to the current validation study. 


\begin{tabular}{|c|c|c|c|c|}
\hline & & \multicolumn{2}{|c|}{ Conventional Microscopy } & \multirow{2}{*}{ Total } \\
\hline & & Hypochromic & Normochromic & \\
\hline \multirow[t]{3}{*}{ Image Recognition Software } & Hypochromic & 111 & 7 & 118 \\
\hline & Normochromic & 6 & 83 & 89 \\
\hline & Total & 117 & 90 & 207 \\
\hline Kappa & 0.8571 & & Level of Agreement & Strong \\
\hline$p$-value & $<0.0001$ & & Expected Agreement & $49.30 \%$ \\
\hline$\%$ Agreement & $92.75 \%$ & & Accuracy & $93.72 \%$ \\
\hline
\end{tabular}

Table 3. Erythrocyte size confusion matrix

\begin{tabular}{|c|c|c|c|c|c|c|}
\hline & & \multicolumn{4}{|c|}{ Conventional Microscopy } & \multirow{2}{*}{ Total } \\
\hline & & Microcytic & Normocytic & Macrocytic & Unknown & \\
\hline \multirow[t]{5}{*}{ Image Recognition Software } & Microcytic & 73 & 10 & 0 & 3 & 86 \\
\hline & Normocytic & 10 & 191 & 2 & 0 & 203 \\
\hline & Macrocytic & 0 & 8 & 38 & 1 & 47 \\
\hline & Unknown & 0 & 0 & 0 & 0 & 0 \\
\hline & Total & 83 & 209 & 40 & 4 & 336 \\
\hline Карра & 0.8141 & & \multicolumn{2}{|c|}{ Level of Agreement } & Strong & \\
\hline$p$-value & $<0.0001$ & & \multicolumn{2}{|c|}{ Expected Agreement } & $45.57 \%$ & \\
\hline$\%$ Agreement & $89.88 \%$ & & \multicolumn{2}{|c|}{ Accuracy } & $89.88 \%$ & \\
\hline
\end{tabular}

Table 4. Erythrocyte shape confusion matrix

\begin{tabular}{|c|c|c|c|c|c|c|c|c|c|c|}
\hline & & \multicolumn{8}{|c|}{ Conventional Microscopy } & \multirow{2}{*}{ Total } \\
\hline & & Normal & Target Cell & Echinocyte & Elliptocyte & Dacrocyte & Spherocyte & Stomatocyte & Unknown & \\
\hline \multirow[t]{9}{*}{ Image Recognition Software } & Normal & 70 & 0 & 0 & 0 & 0 & 2 & 0 & 0 & 72 \\
\hline & Target Cell & 8 & 63 & 0 & 0 & 0 & 0 & 0 & 0 & 71 \\
\hline & Echinocyte & 2 & 0 & 41 & 0 & 0 & 1 & 0 & 0 & 44 \\
\hline & Elliptocyte & 0 & 0 & 1 & 29 & 8 & 1 & 0 & 3 & 42 \\
\hline & Dacrocyte & 3 & 1 & 1 & 5 & 37 & 3 & 0 & 1 & 51 \\
\hline & Spherocyte & 1 & 0 & 0 & 0 & 0 & 61 & 0 & 1 & 63 \\
\hline & Stomatocyte & 7 & 0 & 0 & 0 & 1 & 0 & 62 & 0 & 70 \\
\hline & Unknown & 0 & 0 & 0 & 0 & 0 & 0 & 0 & 0 & 0 \\
\hline & Total & 91 & 64 & 43 & 34 & 46 & 68 & 62 & 5 & 413 \\
\hline Kappa & \multicolumn{2}{|c|}{0.8549} & & & \multicolumn{3}{|c|}{ Level of Agreement } & Strong & & \\
\hline p-value & \multicolumn{2}{|c|}{$<0.0001$} & & & \multicolumn{3}{|c|}{ Expected Agreement } & $14.89 \%$ & & \\
\hline$\%$ Agreement & \multicolumn{2}{|c|}{$87.65 \%$} & & & \multicolumn{3}{|c|}{ Accuracy } & $87.89 \%$ & & \\
\hline
\end{tabular}

A total of 956 erythrocytes from 24 peripheral blood smears were assessed using manual microscopic evaluation and image recognition software. For central pallor, red cell size, and red cell shape, a total of 207, 336 , and 413 cells were tested, respectively. Confusion matrix tables for each red cell morphology classification are listed below (Tables 2, 3 and 4). Interpretation of the kappa values for each classification system showed a "strong" level of agreement between conventional microscopy and the image recognition software. Accuracy for each classification system were $93.72 \%$ for central pallor, $89.88 \%$ for size, and $87.89 \%$ for shape. Cell types identified as "Unknown" are images taken by the software that have low confidence value, insufficient for the machine to provide a definite cell morphology.

Table 5 summarizes the different cell morphologies, from the different classifications systems, that were isolated and identified by the software. Their corresponding sensitivity (true positive rate or recall) and specificity (true negative rate) were computed from the confusion matrices, and 95\% confidence intervals (CI) for the sensitivity and specificity were computed using "exact" Clopper-Pearson confidence intervals.

\begin{tabular}{|c|c|c|c|}
\hline $\begin{array}{c}\text { Red Cell } \\
\text { Classification }\end{array}$ & Morphology & $\begin{array}{c}\text { Sensitivity } \\
{\left[95 \% \mathrm{Cl}^{\circ}\right]}\end{array}$ & $\begin{array}{c}\text { Specificity } \\
{[95 \% \mathrm{Cl}]}\end{array}$ \\
\hline \multirow{5}{*}{ Size } & \multirow{2}{*}{ Microcytic } & $87.95 \%$ & $94.86 \%$ \\
\hline & & [78.96-94.07\%] & [91.37-97.24\%] \\
\hline & Normocytic & $\begin{array}{c}\mathbf{9 1 . 3 9 \%} \\
{[86.73-94.82 \%]}\end{array}$ & $\begin{array}{c}\mathbf{9 0 . 5 5 \%} \\
{[84.08-95.02 \%]}\end{array}$ \\
\hline & \multirow{2}{*}{ Macrocytic } & $95.00 \%$ & $95.95 \%$ \\
\hline & & [83.08-99.39\%] & [94.31-98.60\%] \\
\hline \multirow{4}{*}{ Central Pallor } & \multirow{2}{*}{ Hypochromic } & $94.87 \%$ & $92.22 \%$ \\
\hline & & [84.63-96.82\%] & [89.17-98.10\%] \\
\hline & \multirow{2}{*}{ Normochromic } & $92.22 \%$ & $94.87 \%$ \\
\hline & & [89.17-98.10\%] & [84.63-96.82\%] \\
\hline \multirow{14}{*}{ Shape } & \multirow{2}{*}{ Normal } & $76.92 \%$ & $99.38 \%$ \\
\hline & & [66.91-85.11\%] & [97.77-99.92\%] \\
\hline & \multirow[t]{2}{*}{ Target Cell } & $\mathbf{9 8 . 4 4 \%}$ & $97.71 \%$ \\
\hline & & [91.60-99.96\%] & [95.53-99.01\%] \\
\hline & \multirow{2}{*}{ Dacrocyte } & $80.43 \%$ & 96.19\% \\
\hline & & [66.09-90.64\%] & [93.68-97.90\%] \\
\hline & \multirow{2}{*}{ Elliptocyte } & $85.29 \%$ & $96.57 \%$ \\
\hline & & [68.94-95.05\%] & [94.21-98.16\%] \\
\hline & \multirow{2}{*}{ Spherocyte } & $89.71 \%$ & $99.42 \%$ \\
\hline & & [79.93-95.76\%] & [97.92-99.93\%] \\
\hline & \multirow{2}{*}{ Stomatocyte } & $100 \%$ & $97.72 \%$ \\
\hline & & [94.22-100\%] & [95.96-99.01\%] \\
\hline & \multirow{2}{*}{ Echinocyte } & $95.35 \%$ & 99.19\% \\
\hline & & [84.19-99.43\%] & [97.65-99.83\%] \\
\hline
\end{tabular}




\section{DISCUSSION}

In this study, we evaluated a machine-learning based digital image analysis of red blood cells in peripheral blood smears. Comparison of this system with manual microscopy showed a high kappa value representing a "Strong" level of agreement. The performance characteristics of the system based on its sensitivity and specificity demonstrates its acceptability as an alternative method to the routine blood smear evaluation.

Most of the encountered errors were from the evaluation of red cell shapes, specifically dacrocytes and elliptocytes, due to deviation of the cell from the original ovoid shape of erythrocytes, and, stomatocytes and target cells, due to variation in the features of the central pallor. During the image processing phase, segmentation and cropping of individual cells appear to be incomplete leading to classifying an elliptocyte as dacrocyte and vice versa (Photograph 3). This was reflected in the relatively lower sensitivity values $(80.43 \%$ for dacrocytes and $85.29 \%$ for elliptocytes) of these cell types. For stomatocytes and target cells, the software has a propensity to classify them erroneously as normal erythrocytes (sensitivity of 76.92\%). Overlapping cells were classified by the software into an unknown category as accurate segmentation is required to properly identify abnormal red cell morphology.

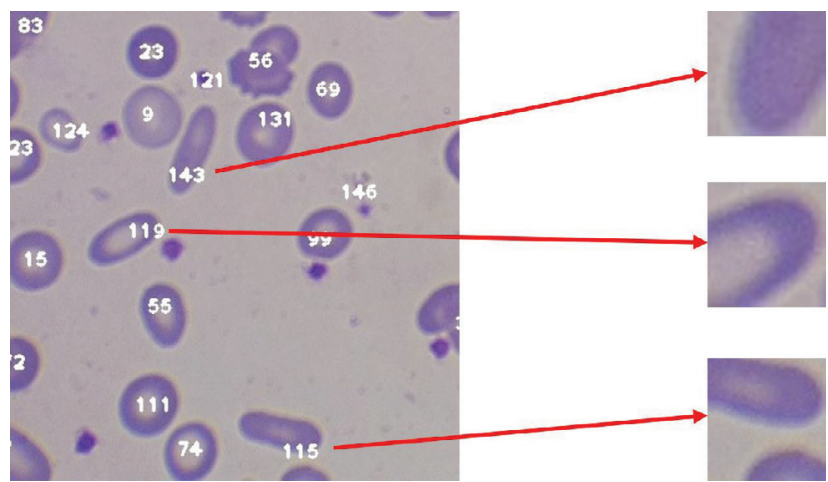

Photograph 3. Incomplete red cell segmentation and cropping after image processing.

The intrinsic processes of a digital image analysis can provide the laboratory several key advantages. Review of digital images can provide timely consultation with experts and inter-institutional referral. These images can be easily stored for future review, education, and training purposes. Machine parameters can also be adjusted to the specific needs of the laboratory in flagging results for further review.

Other published works on erythrocyte classification have focused on classification of erythrocytes into normal or abnormal, ${ }^{21}$ limited number of erythrocyte shapes, ${ }^{22-24}$ or on correlation with specific disease processes based on the combination of extracted features from RBCs. ${ }^{25}$ These studies also demonstrated accuracy rates ranging from $80-91 \%$ while the validated method performed relatively similar with accuracy rates of 87.89-93.72\% depending on the erythrocyte classification. The Cellavision ${ }^{\circledR}$ DM systems Advanced RBC Application (ARBCA) is a commercially available automated digital morphology analyzer that can classify RBCs into 21 categories based on size, shape, color and inclusions. This platform is considered an acceptable screening method prior to definitive classification by a microscopist. ${ }^{26}$ The systems in the aforementioned studies used artificial neural networks in classifying erythrocytes. Our study evaluated a machine-learning system that utilizes support vector machine providing optimum performance characteristics. Support vector machine has been shown to achieve higher accuracy rates from generated classification models after undergoing only a few rounds of training datasets. ${ }^{27}$ Several authors have used this machine-learning method in successfully classifying blasts and other abnormal lymphoid cells in the peripheral blood smear. ${ }^{28,29}$

With the ultimate goal of integration in any diagnostic laboratory setting, the evaluated system used relatively inexpensive components and widely available laboratory equipment in its application. Implementation of the software using a fully-automated and computerized system will require more specialized machines in smear preparation, staining and slide imaging. Several studies and commercially available instruments are tackling these issues with promising, albeit, proprietary technology. ${ }^{26,30}$

\section{CONCLUSION}

We have demonstrated satisfactory performance of a newly-developed and machine-learning based digital image analysis system that is able to satisfactorily identify and classify erythrocytes in blood smears. The image recognition software provides an acceptable diagnostic method in a clinical laboratory setting.

During the conduct of the study, several observations for future implementation and opportunities for research were encountered. Variations in cell morphology secondary to the number of manual processes involved during smear preparation and staining process can be mitigated by using automated systems in smear preparation and staining. Adding more cell classes or features for detection, such as red cell inclusions, and other cell classes, such as leukocytes and platelets will not only provide a more robust software in peripheral blood analysis and refine the image processing. Studies on the effect of the image recognition software on turnaround time and feasibility in a diagnostic laboratory can be undertaken to determine the definite advantages or disadvantages in its utilization.

\section{ACKNOWLEDGEMENTS}

The authors would like to express their utmost appreciation to the section and staff of Hematology of the Department of Laboratories, under the supervisorship of Josephine Z. Ondoy, for their time and expertise during the data gathering process. We would also like to thank the computer engineering students and staff of Mapua University, Paul Daniel C. Divina, John Philip T. Felices, Engr. Carlos C. Hortinela IV and Engr. Jocelyn F. Villaverde for sharing with us their prototype and providing us the much needed technical expertise needed in understanding machine learning and the permission to validate its software. Raspberry Pi is a trademark of the Raspberry Pi Foundation. 


\section{STATEMENT OF AUTHORSHIP}

All authors certified fulfillment of ICMJE authorship criteria.

\section{AUTHOR DISCLOSURE}

The authors declared no conflict of interest.

\section{FUNDING SOURCE}

None.

\section{REFERENCES}

1. Ward PC. The CBC at the turn of the millennium: an overview. Clin Chem. 2000;46(8 Pt 2):1215-20. PMID: 10926915.

2. de Vet HC, Koudstaal J, Kwee WS, Willebrand D, Arends JW. Efforts to improve interobserver agreement in histopathological grading. J Clin Epidemiol. 1995;48(7):869-73. PMID: 7782794. https://doi.org/10.1016/0895-4356(94)00225-f.

3. Ford J. Red blood cell morphology. Int J Lab Hematol. 2013;35(3):351-7. PMID: 23480230. https://doi. org/10.1111/ijlh.12082.

4. Pierre RV. Red cell morphology and the peripheral blood film. Clin Lab Med. 2002;22(1):25-61. PMID: $\quad 11933577$. https;//doi.org/10.1016/s02722712(03)00066-0.

5. Fred HL. Maxwell Myer Wintrobe: new history and a new appreciation. Tex Heart Inst J. 2007;34(3): 328-35. PMID: 17948084. PMCID: PMC1995040.

6. Hur M, CHO JH, Kim H, HONG MH, MOON HW, YUN YM, Kim JQ. Optimization of laboratory workflow in clinical hematology laboratory with reduced manual slide review: comparison between Sysmex XE-2100 and ABX Pentra DX120. Int J Lab Hematol. 2011;33(4):434-40. PMID: 21418541. http://doi.org/10.1111/j.1751-553X.2011.01306.x.

7. Palmer L, Briggs C, McFadden S, et al. CSH recommendations for the standardization of nomenclature and grading of peripheral blood cell morphological features. Int J Lab Hematol. 2015;37(3):287-303. PMID: 25728865. https://doi. org/10.1111/ijlh.12327.

8. Comar SR, Malvezzi M, Pasquini R. Evaluation of criteria of manual blood smear review following automated complete blood counts in a large university hospital. Rev Bras Hematol Hemoter. 2017 Dec;39(4): 306-17. PMID: 29150102. PMCID: PMC5693276. https://doi.org/10.1016/j.bjhh.2017.06.007.

9. Flax S. Why do we still need to evaluate peripheral blood smears? American Association for Clinical Chemistry [Internet]. 2017 Jun [cited 2019 Apr 15]. Available from: https://www.aacc.org/publications/ cln/articles/2017/june/why-do-we-still-need-toevaluate-peripheral-blood-smears.

10. Mohammed EA, Mohamed MM, Far BH, Naugler C. Peripheral blood smear image analysis: a comprehensive review. J Pathol inform. 2014;5(1):9. PMID: 24843821. PMCID: PMC4023032. https://doi. org/10.4103/2153-3539.129442.
11. Kim K, Jeon J, Choi W, Kim P, Ho YS. Automatic cell classification in human's peripheral blood images based on morphological image processing. In: Stumptner M, Corbett D, Brooks M, eds. AI 2001: Advances in Artificial Intelligence. AI 2001. Lecture Notes in Computer Science, vol 2256. Springer, Berlin, Heidelberg. https://doi.org/10.1007/3-540-45656-2_20.

12. Chen S, Zhao M, Wu G, Yao C, Zhang J. Recent advances in morphological cell image analysis. Comput Math Methods Med. 2012;2012. PMID: 22272215. PMCID: PMC3261466. https://doi.org/10.1155/2012/101536.

13. Jordan MI, Mitchell TM2. Machine learning: trends, perspectives, and prospects. Science. 2015;349(6245):255-60. PMID: 26185243. https://doi. org/10.1126/science.aaa8415.

14. Cabitza F, Banfi G. Machine learning in laboratory medicine: waiting for the flood?. Clin Chem Lab Med. 2018;56(4):516-24. PMID: 29055936. https://doi. org/10.1515/cclm-2017-0287.

15. Gunčar G, Kukar M, Notar M, et al. An application of machine learning to haematological diagnosis. Sci Rep. 2018;8(1):411. PMID: 29323142. PMCID: PMC5765139. https://doi.org/10.1038/s41598-01718564-8.

16. Ghosh S, Roy A, Sarkar D. Assessment of morphologically altered rbcs using image processing tools. Materials today: proceedings. 2016;3(10 part A): 3361-6. https://doi.org/10.1016/j.matpr.2016.10.017.

17. Kraus OZ, Grys BT, Ba J, et al. Automated analysis of high-content microscopy data with deep learning. Mol Syst Biol. 2017;13(4):924. PMID: 28420678. PMCID: PMC5408780. https://doi.org/10.15252/msb.20177551.

18. Adollah R, Mashor MY, Nasir NM, Rosline H, Mahsin $\mathrm{H}$, Adilah H. Blood cell image segmentation: a review. In: 4th Kuala Lumpur international conference on biomedical engineering. Springer, Berlin, Heidelberg; 2008, pp. 141-144.

19. Divina P, Felices J. Identification of abnormal red blood cells and diagnosing specific types of anemia using image processing and support vector machine. Mapua University, Philippines. Forthcoming 2019.

20. McHugh ML. Interrater reliability: the kappa statistic. Biochem Med (Zagreb). 2012;22(3):276-82. PMID: 23092060. PMCID: PMC3900052.

21. Tomari R, Zakaria WN, Jamil MM, Nor FM, Fuad NF. Computer aided system for red blood cell classification in blood smear image. Procedia Comput Sci. 2014;42: 206-13. https://doi.org/10.1016/j.procs.2014.11.053.

22. Dalvi PT, Vernekar N. Computer aided detection of abnormal red blood cells. 2016 IEEE International Conference on Recent Trends in Electronics, Information \& Communication Technology (RTEICT), Bangalore, India; 2016, pp. 1741-6.

23. Syahputra MF, Sari, AR, Rahmat RF. Abnormality classification on the shape of red blood cells using radial basis function network. 2017 4th International Conference on Computer Applications and Information Processing Technology (CAIPT), Kuta Bali; 2017, pp. 1-5.

24. Lee H, Chen YP. Cell morphology based classification for red cells in blood smear images. Pattern Recognit Lett. 2014;49:155-61. https://doi. org/10.1016/j.patrec. 2014.06.010 
25. Kim G, Jo Y, Cho H, Min HS, Park Y. Learning-based screening of hematologic disorders using quantitative phase imaging of individual red blood cells. Biosensors and Bioelectronics. 2019;123:69-76. PMID: 30321758. https;/doi.org/10.1016/j.bios.2018.09.068.

26. Kratz A, Lee SH, Zini G, et al. Digital morphology analyzers in hematology: ICSH review and recommendations. International journal of laboratory hematology. 2019;41(4):437-47. PMID: 31046197. https://doi.org/10.1111/ijlh.13042.

27. Barghout L. Spatial-taxon information granules as used in iterative fuzzy-decision-making for image segmentation. In: Pedrycz W., Chen SM. (eds) Granular Computing and Decision-Making. Studies in Big Data, vol 10. Springer, Cham; 2015. https://doi. org/10.1007/978-3-319-16829-6_12.
28. Bigorra L, Merino A, Alférez S, Rodellar J. Feature analysis and automatic identification of leukemic lineage blast cells and reactive lymphoid cells from peripheral blood cell images. J Clin Laboratory Anal. 2017;31(2):e22024. https://doi.org/10.1002/jcla.22024.

29. Rodellar J, Alférez S, Acevedo A, Molina A, Merino A. Image processing and machine learning in the morphological analysis of blood cells. Int J Lab Hematol. 2018;40(Suppl 1):46-53. PMID: 29741258. htpps://doi.org/10.1111/ijlh.12818.

30. Winkelman JW, Tanasijevic MJ, Zahniser DJ. A novel automated slide-based technology for visualization, counting, and characterization of the formed elements of blood: a proof of concept study. Arch Pathol \& Lab Med. 2017;141(8):1107-12. PMID: 28421831. https://doi.org/10.5858/arpa.2016-0633-OA.

Disclaimer: This journal is OPEN ACCESS, providing immediate access to its content on the principle that making research freely available to the public supports a greater global exchange of knowledge. As a requirement for submission to the PJP, all authors have accomplished an AUTHOR FORM, which declares that the ICMJE criteria for authorship have been met by each author listed, that the article represents original material, has not been published, accepted for publication in other journals, or concurrently submitted to other journals, and that all funding and conflicts of interest have been declared. Consent forms have been secured for the publication of information about patients or cases; otherwise, authors have declared that all means have been exhausted for securing consent.

\section{Publish in the new PJP. Visit our website: htp://philippinejournalofpathology.org}

